\title{
CARACTERIZACIÓN TÉRMICA DE LA CAPA ACTIVA DE UN GLACIAR ROCOSO EN MEDIO PERIGLACIAR DE ALTA MONTAÑA MEDITERRÁNEA. EL EJEMPLO DEL CORRAL DEL VELETA (SIERRA NEVADA, ESPAÑA)
}

\author{
F. SALVADOR FRANCH ${ }^{(1,3)}$ * A. GÓMEZ ORTIZ ${ }^{(1,3)}$ \\ M. SALVÀ CATARINEU ${ }^{(1)}$, D. PALACIOS ESTREMERA ${ }^{(2,3)}$ \\ ${ }^{1}$ Departament de Geografia Física i Anàlisi Geogràfica Regional. Universitat de Barcelona \\ ${ }^{2}$ Departamento de Análisis Geográfico Regional y Geografía Física. \\ Universidad Complutense de Madrid \\ ${ }^{3}$ Grupo de Investigación Paisaje y Paleoambientes en Montaña Mediterránea
}

\begin{abstract}
RESUMEN. Se analizan los datos de la temperatura del interior del suelo (capa activa) del periodo 1998-99/2008-09 de un glaciar rocoso incipiente en el Corral del Veleta (Sierra Nevada) con masas heladas en su interior en proceso de degradación. El control térmico alcanza los $150 \mathrm{~cm}$ y corresponde a los niveles de $-5,-20,-50,-100$ y $-150 \mathrm{~cm}$. Los registros obtenidos, una vez depurados y tratados estadísticamente, vienen a señalar un comportamiento de las temperaturas que sigue el siguiente patrón: un periodo frío muy largo (invierno) de valores negativos, un periodo relativamente corto de temperaturas moderadas (verano) y otros dos episodios muy cortos distanciados en el tiempo en los que la temperatura cambia de signo: de positiva a negativa (otoño) y de negativa a positiva (verano). El régimen térmico del verano, que propicia la llegada de la onda expansiva externa al techo de las masas heladas subyacentes, facilita la degradación de éstas y, al tiempo, colapsos del manto detrítico.
\end{abstract}

Thermal pattern in the active layer of a rock glacier in the Mediterranean high mountain (Corral del Veleta, Sierra Nevada, Spain)

ABSTRACT. The analysis of temperature data inside the soil (active layer), for the period 1998-1999/2008-2009, in an incipient rock glacier in Corral del Veleta cirque (Sierra Nevada) with presence of frozen masses in degradation process inside of it, is submitted. Thermal control covers $150 \mathrm{~cm}$ and data is collected at $-5,-20,-50,-100$ and $-150 \mathrm{~cm}$. This data, refined and statistically worked, shows this pattern: a very long cold period (winter) with negative values, a relatively short period with moderate temperatures (summer) and two very short periods when temperature changes form positive values to negative values (autumn) and from negative values to positive values (summer). The summer thermal pattern causes the arrival of the external shock wave at the top of the frozen underlying masses, facilitates the degradation of these underlying masses, and collapses the detrital stratum. 
Palabras clave: capa activa, corral del Veleta (Sierra Nevada), degradación, glaciar rocoso, masas heladas, onda térmica, régimen térmico.

Key words: active layer, corral del Veleta (Sierra Nevada), degradation, rock glacier, frozen masses, thermal wave, thermal pattern.

Enviado el 28 de octubre de 2010

Aceptado el 3 de febrero de 2011

*Correspondencia: Departament de Geografia Física i Anàlisi Geográfica Regional. Facultat de Geografia i Història. Universitat de Barcelona. c/ Montalegre, 6, 08001-Barcelona. E-mail: fsalvador@ub.edu

\section{Introducción}

El desarrollo de estudios acerca del comportamiento térmico del suelo en medios fríos se inició a finales del siglo XIX en Canadá, habiendo tenido continuidad durante las décadas siguientes (Callendar, 1897). En la segunda mitad del siglo XX, gracias al avance tecnológico en instrumentación de recogida de datos con aparatos autónomos, los estudios se trasladaron también a los Alpes suizos (Haeberli, 1973; King, 1990; Hoelzle et al., 1999; Delaloye, 2004), regiones nórdicas europeas y siberianas y a la Antártida, en este último caso al amparo de los programas de investigación internacionales sobre este continente helado (Ramos y Vieira 2004). Recientemente, también ha venido preocupando el comportamiento térmico del suelo en las montañas tropicales, en particular en los estratovolcanes mexicanos y peruanos que aún mantienen glaciares y permafrost (Andrés de Pablo et al., 2010a, 2010b; Palacios et al., 2009), así como en los Andes de Mendoza (Carrera Gómez et al., 2008)

Por lo que respecta a las montañas de la Península Ibérica y desde la perspectiva geomorfológica, las primeras publicaciones podrían corresponder a finales de 1980 (Ramos et al.,1998). Desde entonces resultan numerosas y se reparten, prácticamente, por todos los niveles de cumbres afectados por procesos crionivales y/o periglaciares (Pirineos, Picos de Europa, montañas leonesas y gallegas, Sistema Central, Sierra Nevada, etc.). Todos estos trabajos participan de objetivos comunes que son el determinar el ritmo térmico de la superficie e interior del suelo (ciclos de hielo-deshielo en los niveles externos del suelo y periodos de engelamiento y deshielo en sus niveles internos). Casi siempre con la finalidad de explicar procesos morfogénicos y modelados asociados en el contexto geográfico particular de la montaña estudiada. La acumulación de datos hasta ahora viene a mostrar comportamientos térmicos diferenciados entre enclaves montañosos e incluso dentro del mismo macizo. En tal sentido, subrayar que altitud, orientación, duración de la nieve en el suelo, presencia-ausencia de cuerpos helados en el seno del suelo, naturaleza y composición de la fracción órgano-mineral del suelo, etc. resultan determinantes en las variaciones que sufre la temperatura cuando incide en la superficie e interior del suelo (Serrano et al., 2006, 2010; Andrés de Pablo y Palacios 2010c; Marcos y Palacios 2004; Santos González et al., 2009; Chueca y Julian, 2004; Vieira et al., 2003; Salvador Franch et al., 2010; Oliva y Gómez, 2004). 


\section{Objeto de estudio}

Sierra Nevada, por encima de los $2625 \mathrm{~m}$, goza de un ambiente crionival de montaña mediterránea, caracterizado por la predominancia de los procesos morfogénicos fríos con repercusión en las formas de modelado. Estos procesos están asociados al comportamiento de la acción mecánica del hielo, la nieve, el viento y las aguas de fusión nival, principalmente. Este ambiente crionival tuvo un desarrollo altitudinal más extenso durante la Pequeña Edad del Hielo con existencia, incluso, de pequeños focos glaciares arrinconados
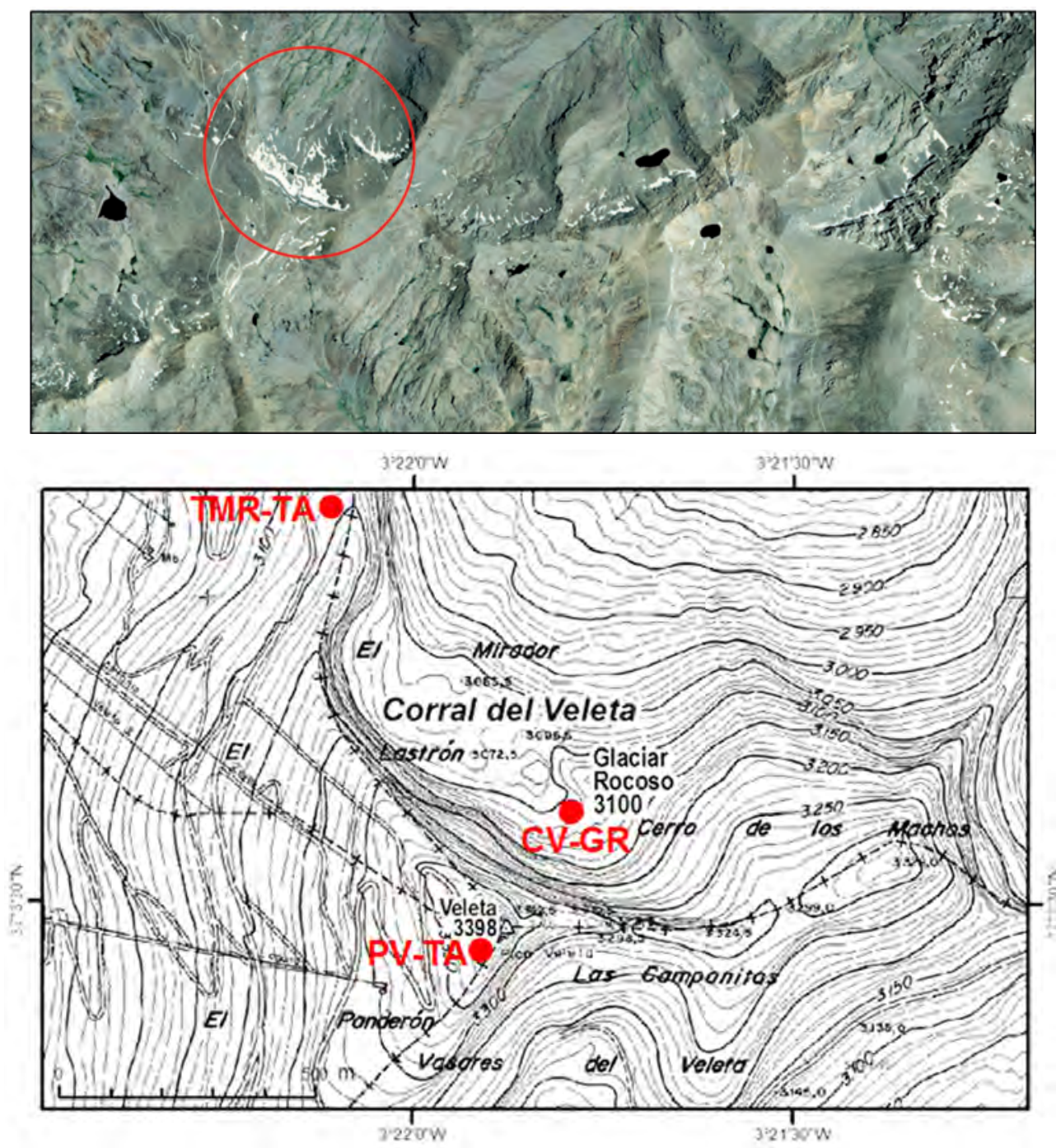

Figura 1. Sector de cumbres de Sierra Nevada (tramo Veleta-Mulhacén). El círculo indica la situación del Corral del Veleta. En detalle, topografía del Corral del Veleta y localización de los puntos de muestreo térmico (TMR-TA, PV-TA, CV-GR). 


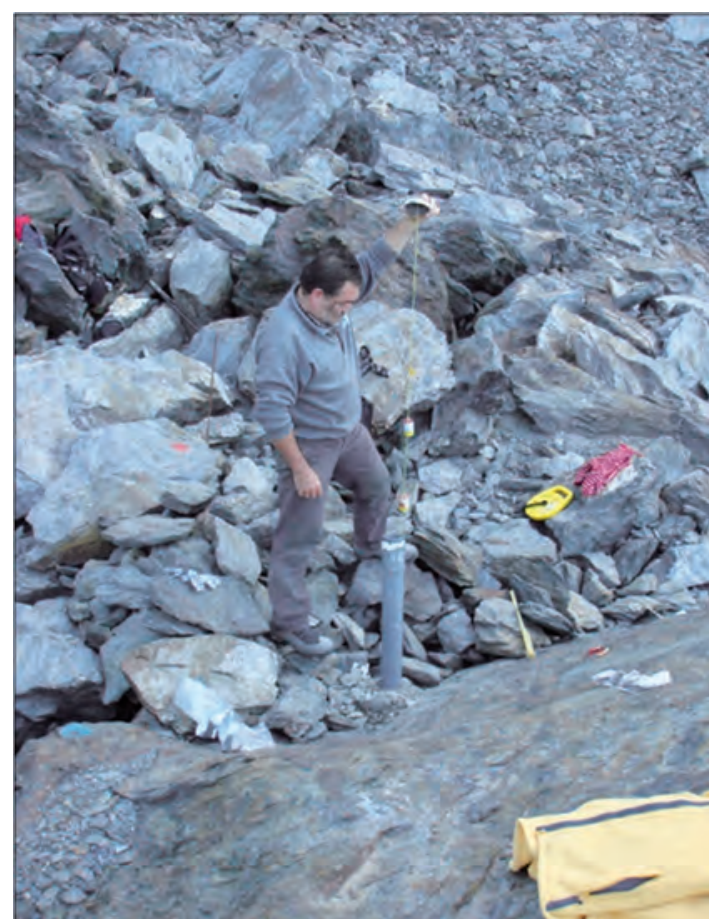

Figura 2. Colocación de la cadena de sensores térmicos en el seno del glaciar rocoso.

en el interior de los antiguos circos cuaternarios, tal como se afirma en la documentación de época y los registros naturales identificados (Gómez Ortiz et al., 2009a, 2009b). El último de estos focos en persistir fue el del Corral del Veleta, que aún guarda restos de masas heladas (hielos glaciares y permafrost) enterradas bajo manto de bloques (Fig. 1).

El objeto de estudio de este trabajo se centra en el seguimiento del comportamiento térmico del suelo de un pequeño glaciar rocoso activo instalado en el tercio oriental del Corral del Veleta en el que perduran restos de las masas heladas citadas (Fig. 2). Se propone como objetivo preciso conocer el ritmo térmico de los valores alcanzados en la capa activa y determinar su influencia en la degradación que viene afectando el techo de dicha masa helada y/o permafrost, así como establecer el periodo temporal en que ello sucede.

\section{Método de estudio e instrumentación utilizada}

El registro de datos térmicos del glaciar rocoso del Corral del Veleta se inició en 1999 a partir de sensores de alimentación autónoma y registro continuo (data logger) tipo TINYTALK II (rango $-35 \mathrm{a}+70^{\circ} \mathrm{C}$, precisión $\pm 0,35^{\circ} \mathrm{C}$ ) (Ramos et al., 1998, 2002), siendo sustituidos en 2002 por otros de tipo UTL-1 (rango -30 a $+40^{\circ} \mathrm{C}$, precisión $\pm 0,1^{\circ} \mathrm{C}$ ) (Hoelzle et al., 1999). Los niveles del suelo muestreados fueron inicialmente $-2,-15,-40,-90$ y $-190 \mathrm{~cm}$, pasando luego (agosto 2005) a $-5,-20,-50,-100 \mathrm{y}-150 \mathrm{~cm}$, ajustándolos, de esta forma, al protocolo establecido en otros enclaves de Sierra Nevada 
y Pirineo Oriental. La frecuencia de observación ha oscilado entre 4'48 h y 1 h, pero en la actualidad (desde agosto 2006) es de 2 h lo que asegura que el instrumental funcione de manera continua durante casi dos años. La temperatura del aire, como serie de referencia, se ha registrado en las inmediaciones del glaciar rocoso. Primero, en una estación automática propia (TMR, torre meteorológica de referencia) situada en la Loma del Veleta, a 3098 m y, a partir de 2003, en el Pico del Veleta (estación PV-TA, 3398 m).

Hasta la fecha se dispone de información térmica del suelo (estación CV-GR, $3107 \mathrm{~m}$ ) y del aire procedente de 10 campañas anuales (de septiembre a agosto, 199899/2008-09) (Tabla 1). El volumen anual de datos útiles obtenidos supera habitualmente el $90 \%$ respecto al máximo posible, excepto las dos primeras campañas que se iniciaron en noviembre. Solo faltan completos los datos de 2000-01, pues la acumulación de nieve en el lugar de muestreo impidió la recuperación de datos.

A partir de los registros térmicos generados, depurados y tratados estadísticamente, se han obtenido los datos diarios medios y extremos utilizados para la elaboración de los valores promedio de la serie disponible (mensuales, estacionales y anuales), sintetizados en las Tablas 2-6 y Figuras 4-11. En las referidas tablas y figuras se han unificado las profundidades controladas al modelo de los últimos años $(-5,-20,-50,-100$ y $-150 \mathrm{~cm})$. La temperatura del aire de los tres primeros años (TMR) se ha ajustado a la cota correspondiente al Pico del Veleta (PV-TA).

\section{4. Área de estudio y contexto geomorfológico}

En cuanto al marco geográfico particular en el que se ha llevado a cabo este seguimiento térmico de la capa activa se sitúa en la cabecera del valle del Guarnón, antiguo circo glaciar cuaternario, en su repisa más elevada, en el denominado Corral del Veleta (>3100 m). Este paraje mantiene orientación NE y está bien encarado a los flujos húmedos atlánticos. Se trata de un cuenco encerrado por altas paredes y aislado del valle por un cordón morrénico frontolateral. El surco que define su interior está colmado de bloques y gruesos cantos procedentes de la destrucción de los tajos que lo delimitan. Este caos de material clástico conforma diferentes modelados y de entre ellos el más significativo es su activo talud detrítico y, sobre todo, el incipiente glaciar rocoso instalado en su tercio oriental, objeto del presente seguimiento térmico (Fig. 3). El glaciar rocoso, asentado sobre masas heladas, tiene una longitud de $109 \mathrm{~m}$ y una anchura media de $32 \mathrm{~m}$ Se orienta, primero, de S a N, y luego de W a E. En su inicio, el manto de bloques que lo conforma, se dispone en coladas pero después, y de acuerdo con la pendiente, se transforman en arcos sucesivos para, finalmente, fusionarse. Las masas heladas que incluye en su interior se localizaban a partir de $120 \mathrm{~cm}$ de profundidad en 1999; en la actualidad, con seguridad por debajo de los $2 \mathrm{~m}$. Climáticamente hay un hecho significativo que define al Corral del Veleta y es la larga duración de la nieve en el suelo que tiende a perdurar, de manera continua, desde mediados de noviembre hasta mediados de junio-julio. Sin duda, la configuración morfotopográfica del Corral, su favorable orientación a los flujos húmedos atlánticos, los aludes y el barrido del viento sobre las lomas limítrofes, son factores que favorecen la permanencia de la nieve en este reducto. 


\begin{tabular}{|c|c|c|c|c|c|c|c|c|c|}
\hline 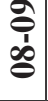 & 肖 & & & $x$ & $x$ & $x$ & $x$ & $x$ & $x$ \\
\hline 今̊ & 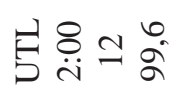 & & & $\frac{\partial}{\infty}$ & $x$ & $x$ & $x$ & $x$ & $x$ \\
\hline کे & 巨હ & & & $x$ & $x$ & $x$ & $x$ & $\frac{\partial}{\infty}$ & $\frac{\nabla}{\omega}$ \\
\hline $\begin{array}{l}\text { ๖̊ } \\
\text { ம் }\end{array}$ & 巨艺○ & & & $\frac{D}{\infty}$ & $x$ & $x$ & $x$ & $x$ & $x$ \\
\hline & & & & 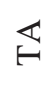 & $n$ & $\stackrel{\Upsilon}{\uparrow}$ & $\stackrel{\cap}{i}$ & $\underset{1}{8}$ & $\frac{\circ}{7}$ \\
\hline $\begin{array}{l}n \\
\stackrel{1}{1} \\
\dot{d}\end{array}$ & 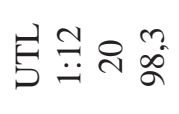 & & & $x$ & $x$ & $x$ & $\frac{\partial}{\infty}$ & $x$ & $\frac{\partial}{m}$ \\
\hline હें & 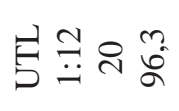 & & & $x$ & $\frac{\partial}{\infty}$ & $x$ & $\frac{\partial}{\infty}$ & $x$ & $\frac{\nabla}{m}$ \\
\hline $\begin{array}{c}\text { ֻุ่ } \\
\text { ฮั }\end{array}$ & 卢导 & & $\frac{D}{\infty}$ & & $\frac{\pi}{\infty}$ & $\frac{\Gamma}{\infty}$ & $x$ & $x$ & $x$ \\
\hline ํํㅇ & 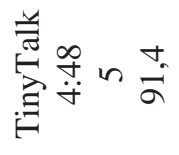 & & $x$ & & $\frac{\vec{T}}{\infty}$ & $x$ & $x$ & $\frac{\nabla}{\infty}$ & $x$ \\
\hline $\begin{array}{l}\text { ह̊ } \\
\text { ̊̀ }\end{array}$ & 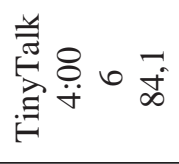 & & $x$ & & $x$ & $x$ & $x$ & $x$ & $x$ \\
\hline वे & 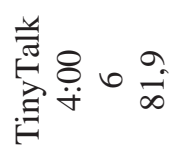 & & $x$ & & $x$ & $x$ & $x$ & $\frac{\partial}{\infty}$ & $\frac{\partial}{\infty}$ \\
\hline | & 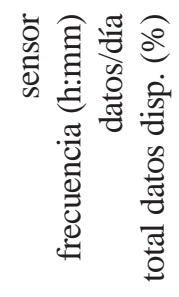 & 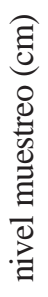 & 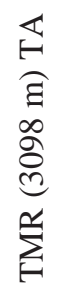 & 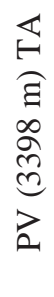 & 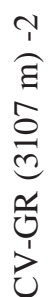 & $\frac{n}{1}$ & $\underset{1}{+}$ & @i & $\frac{8}{1}$ \\
\hline
\end{tabular}




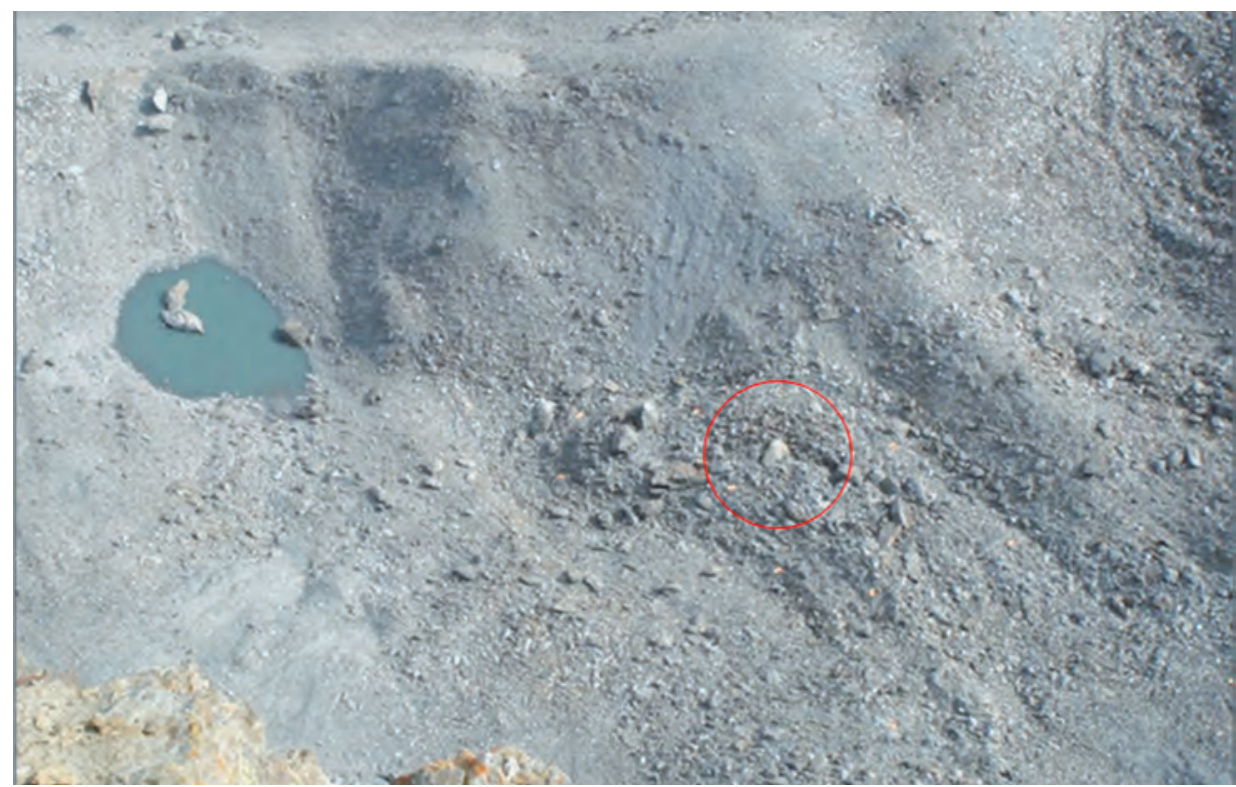

Figura 3. Sector oriental del Corral del Veleta. Identificación del glaciar rocoso y del sector de muestreo térmico.

\section{Controles efectuados y resultados obtenidos}

\subsection{Temperatura media (Tx)}

La Tabla 2 y Figura 4 muestran que la temperatura media (Tx) anual del aire se fija en los $-0,4^{\circ} \mathrm{C}$, manteniéndose sólo positiva durante el verano $\left(8,1^{\circ} \mathrm{C}\right)$ pues en otoño, incluso no supera el umbral de los $0^{\circ} \mathrm{C}$. La amplitud media anual, a partir de las medias mensuales, se instala en los $16,2^{\circ} \mathrm{C}$. Por lo que respecta al suelo la temperatura media tiende a decrecer fijándose en $0,6^{\circ} \mathrm{C}$ en superficie $(-5 \mathrm{~cm})$ y los $-1,4^{\circ} \mathrm{C}$ en el nivel más profundo $(-150 \mathrm{~cm})$. Sin embargo, su distribución estacional presenta contrastes acusados. Invierno y primavera se mantiene negativa, frente al verano y otoño que resulta positiva, a excepción del nivel de $-150 \mathrm{~cm}$ que todo el año refleja valor negativo (entre $-0,5^{\circ} \mathrm{C}$ y $-2,3^{\circ} \mathrm{C}$ ).

El reparto térmico mensual en el suelo se muestra variable, aunque imperando los valores negativos. La mayor amplitud térmica se registra a $-5 \mathrm{~cm}$, que se fija en $17,6^{\circ} \mathrm{C}$ (enero $-5,4^{\circ} \mathrm{C}$, agosto $12,2^{\circ} \mathrm{C}$ ). La menor a $-150 \mathrm{~cm}, 3,1^{\circ} \mathrm{C}$ (septiembre $0,1^{\circ} \mathrm{C}$, marzo $-3,0^{\circ} \mathrm{C}$ ). De noviembre a abril los valores de la Tx permanecen por debajo de los $0^{\circ} \mathrm{C}$ en todos los niveles, escalonadamente más fríos en superficie que en profundidad, incrementando su valor negativo hasta febrero en superficie $(-5,-20 \mathrm{~cm})$ y marzo en profundidad, iniciándose, después, su recuperación. Julio y agosto son los meses más cálidos, invirtiéndose el escalonamiento térmico, ahora con temperaturas más elevadas en superficie que en profundidad $\left(>11^{\circ} \mathrm{C}\right)$ debido, siempre, a la mayor eficacia de la 


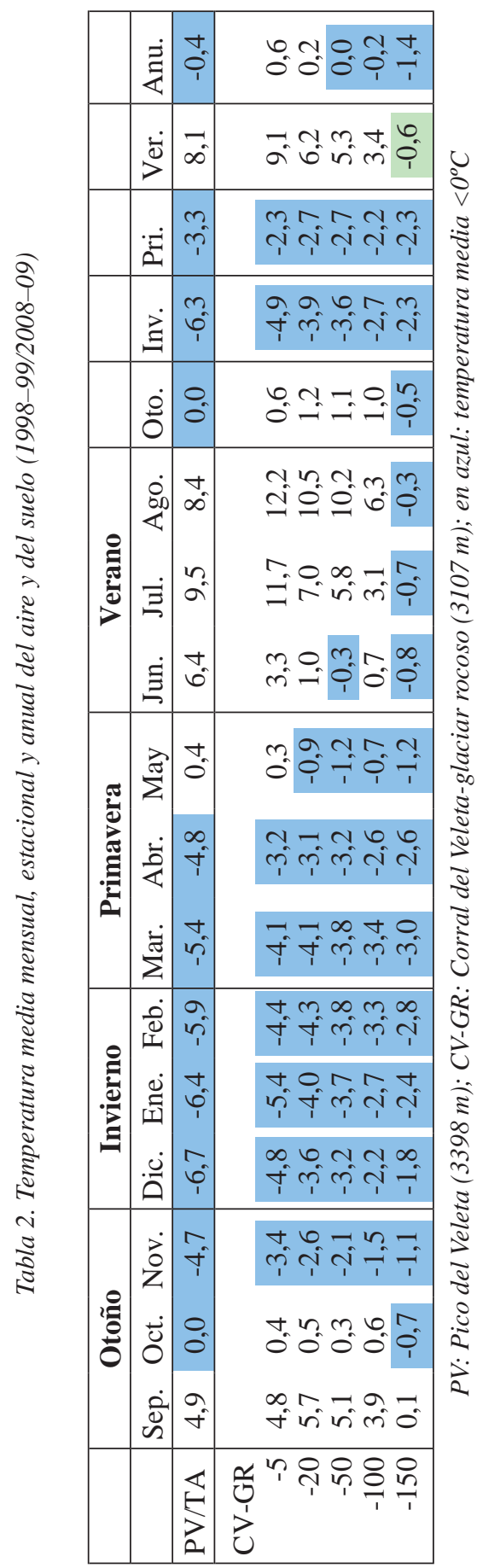




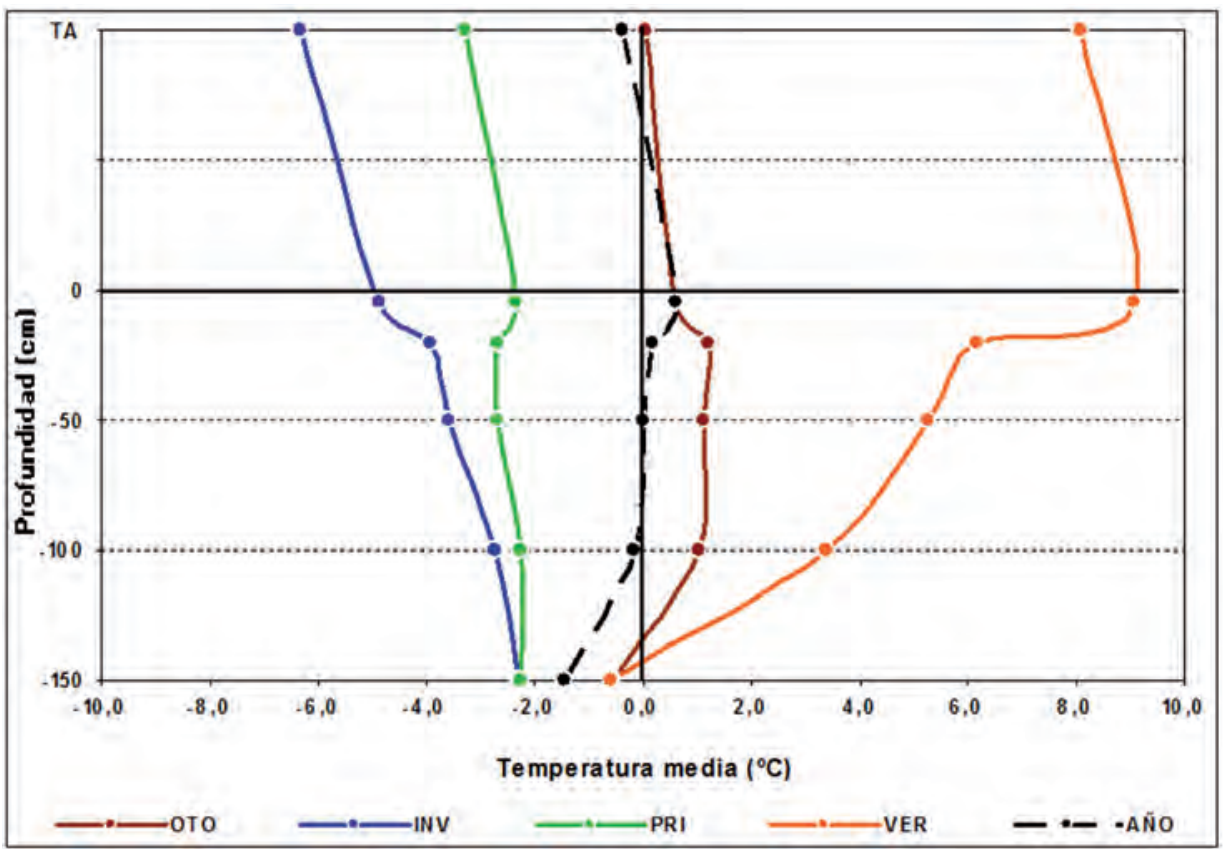

Figura 4. Temperatura media estacional y anual del aire y del suelo (1998-99/2008-09).

influencia térmica del aire. Mayo-junio y septiembre-octubre son episodios de tránsito entre ambas situaciones con valores extremos de Tx, cálidos o fríos respectivamente, sobre todo en los niveles intermedios del suelo (entre -20 y $-100 \mathrm{~cm}$ ). El nivel más profundo tiende a mantener valores negativos, hasta $-3^{\circ} \mathrm{C}$, aunque en septiembre ya están cercanos a $\operatorname{los} 0^{\circ} \mathrm{C}$ o, incluso, ligeramente positivos $\left(0,1^{\circ} \mathrm{C}\right)$.

En cuanto al ritmo térmico anual diario del suelo (Figs. 5 y 6) hay que resaltar que responde a un patrón de conducta temporal repetitivo (Salvador Franch et al, 2010). Ambas figuras, que muestran la secuencia octubre 2005-agosto 2009, reflejan: un largo periodo frío de temperaturas negativas, un corto periodo de temperaturas positivas moderadas-cálidas y el tránsito entre ambos, organizado en dos periodos distanciados en el tiempo en los que los valores registrados tienden a instalarse por encima de los cero grados o por debajo de ellos. Sin embargo, este reparto temporal de valores térmicos no siempre mantiene igual ritmo ni alcanza las mismas profundidades en el suelo, como así sucede, por ejemplo, durante el periodo frío de los años analizados. Por ejemplo, el 2008-09 resultó menos frío que los anteriores (2005-06, 2006-07 y 2007-08). Y entre éstos últimos existieron diferencias en cuanto al alcance de temperaturas máximas negativas en el seno del suelo (Fig. 6). En tal sentido, señalar que a lo largo de los periodos fríos de los años 2005-06, 2006-07 y 2007-08 éstas se situaron entre los $-3,7^{\circ} \mathrm{C}$ y los $-4,7^{\circ} \mathrm{C}$ a los $-150 \mathrm{~cm}$, mientras que en el 2008-09, a la misma profundidad, se instalaron en torno a los $-1,6^{\circ} \mathrm{C}$. 


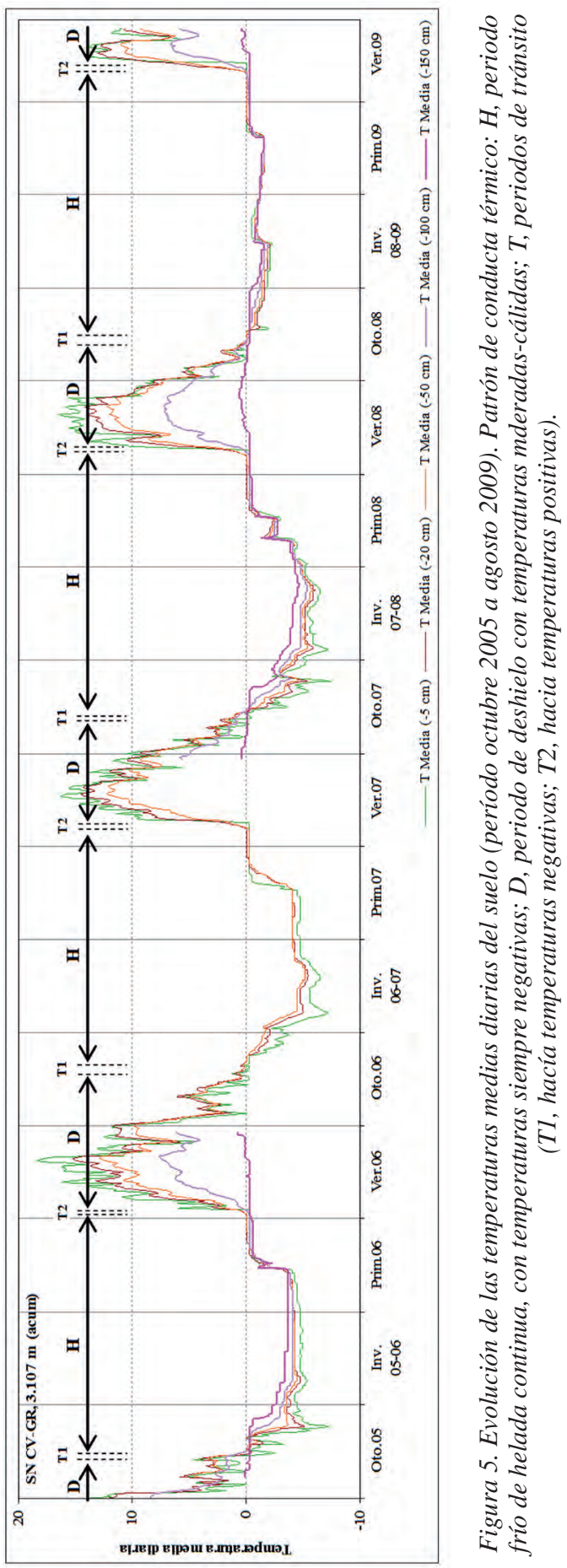

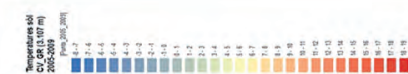

$600 z / 80 / 12$
$600 z / 80 / 10$

$6002120 / 10$

600z/90/10

$6002 / 50 / 10$

$6002 / 70 / 10$

6002/80/10

600z/20/10

6002110/10

800ะルL/LO

$800 z / L L / 10$

800z/0L/10

$8002 / 60 / 10$

$800 z / 80 / 10$

$800 \pi / 20 / 10$

$8002 / 90 / 10$

8002/so/10

800z/to/10

800ट/ع0/เ

800zл20/10

8002110/10

LOOZ/ZL/LO

LOOZ/LL/LO

Looz/ot/10

LOOZ/60/10

LOOZ/80/เO

LO0Z/LO/LO

LOOz/90/10

LOOZ/SO/10

LOOZ/to/lo

L00z/80/10

LOoz/zo/LO

L00Z/10/10

900zスZL/10

900z/Lᄂ/10

900z/0L/Lo

900z/60/10

900z/80/10

9002/LO/LO

900z/90/ 10

900z/so/lo

900z/to/lo

900ะ/ะ/

900z/zo/1

900zлL/L

s002/ZL/10

s00z/LL/LO

s002/0L/10

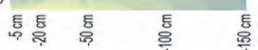

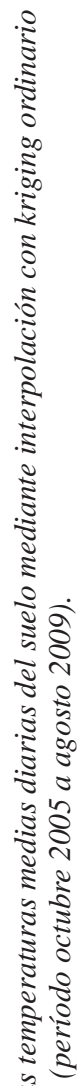

s

$\approx$

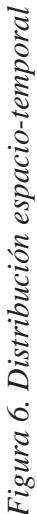




\subsection{Temperaturas extremas (Mex, mex) y amplitud térmica}

La distribución de los valores térmicos extremos o absolutos se refleja en la Tablas 3 y 4 , de cuyos datos pueden obtenerse las respectivas amplitudes térmicas en cada uno de los niveles controlados y su reflejo en el gráfico estacional (Fig. 7). El comportamiento anual denota un decrecimiento progresivo entre el aire y las capas más internas del suelo, lo que vendría a confirmar la dificultad que debe encontrar la expansión de la onda de radiación externa en el seno del suelo.

Tabla 3. Temperaturas y amplitudes extremas

\begin{tabular}{|r|c|c|c|}
\hline niveles de muestreo & Mex & mex & Atex \\
\hline Aire (TA) & 28,6 & $-27,8$ & 56,4 \\
\hline Suelo $-5 \mathrm{~cm}$ & 28,6 & $-19,6$ & 48,2 \\
$-20 \mathrm{~cm}$ & 28,9 & $-12,3$ & 41,2 \\
$-50 \mathrm{~cm}$ & 15,7 & $-12,9$ & 21,6 \\
$-100 \mathrm{~cm}$ & 10,6 & $-6,3$ & 16,9 \\
$-150 \mathrm{~cm}$ & 0,7 & $-4,8$ & 5,5 \\
\hline
\end{tabular}

Mex: Máxima extrema absoluta; mex: mínima extrema absoluta; Atex: amplitud térmica extrema.

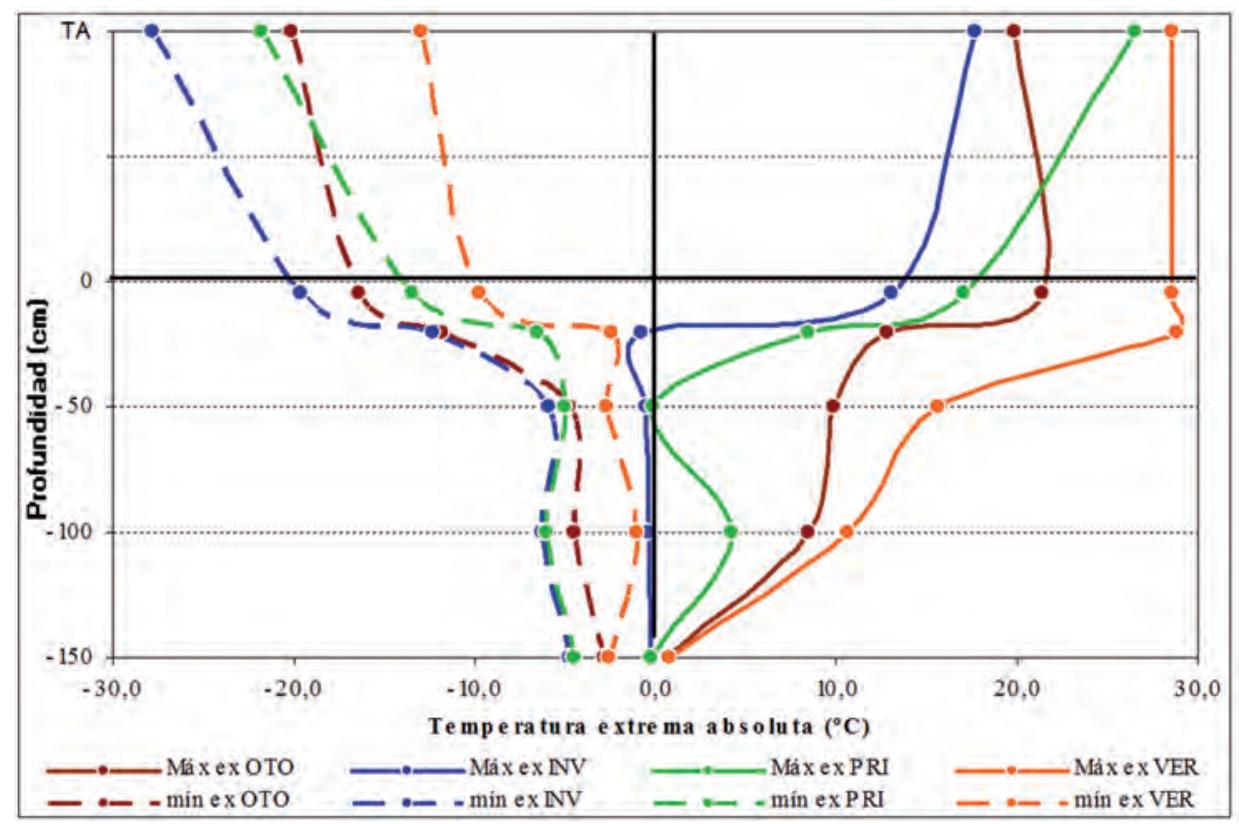

Figura 7. Temperaturas extremas absolutas estacionales del aire y del suelo (1998-99/2008-09). 


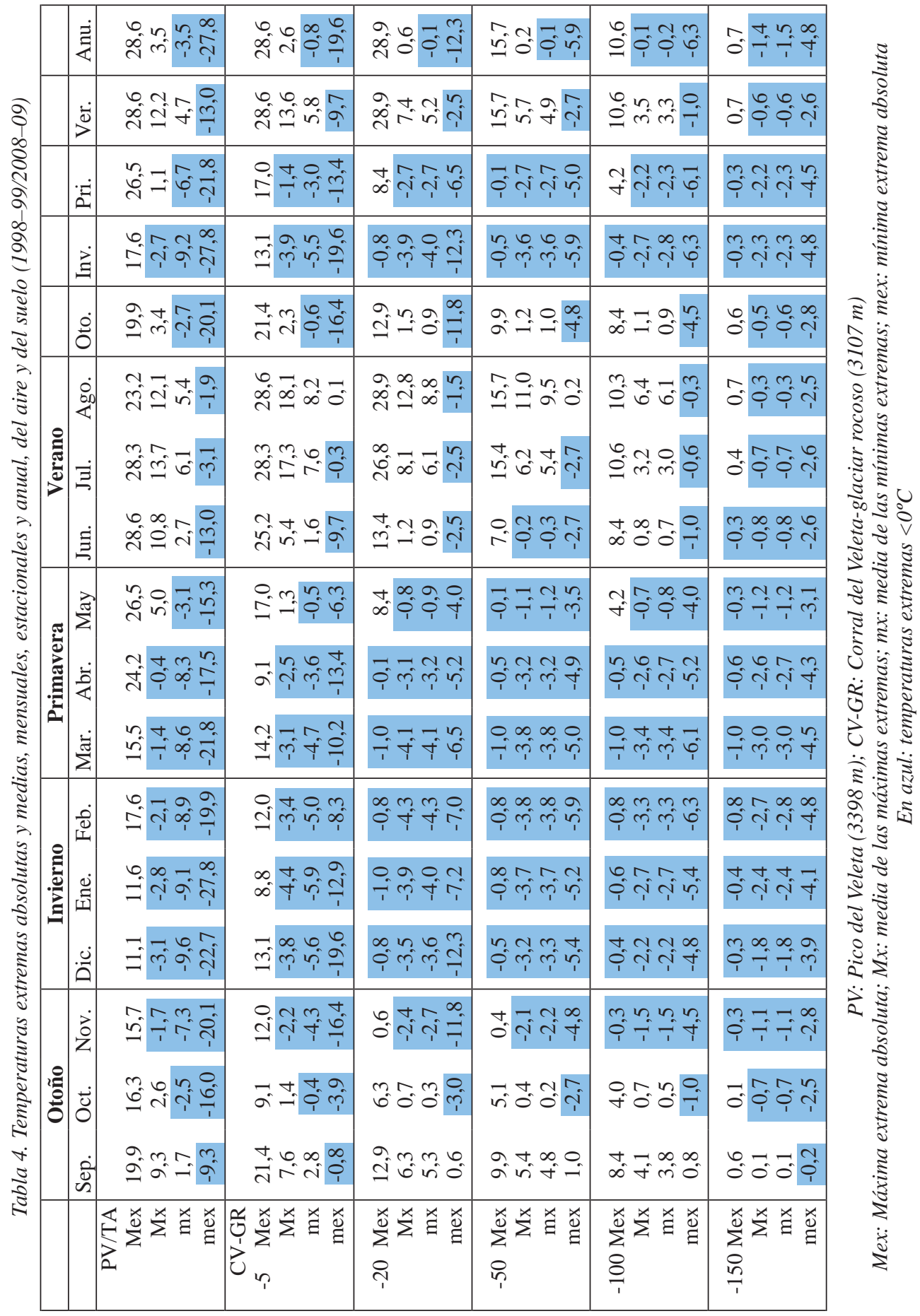


Los valores extremos mínimos y, por consiguiente, las menores amplitudes térmicas se establecen a partir de los $-50 \mathrm{~cm}$ y se consolidan a partir de $10 \mathrm{~s}-100 \mathrm{~cm}$ y por debajo de ellos. Este hecho se refleja en el balance negativo que ofrece la diferencia térmica entre las medias de las máximas extremas $(\mathrm{Mx})$ y la medias de las mínimas extremas $(\mathrm{mx})$, siendo ambas negativas: $-0,1^{\circ} \mathrm{C}, \mathrm{a}-100 \mathrm{~cm}$ y $-0,1^{\circ} \mathrm{C}$ a $-150 \mathrm{~cm}$ (Tabla 4$)$. Este dato podría inducir a establecer la amplitud de $0^{\circ} \mathrm{C}$ ligeramente por debajo de este último nivel del suelo, a pesar de que puntualmente a $-150 \mathrm{~cm}$ los valores tiendan a situarse tímidamente por encima de los $0^{\circ} \mathrm{C}\left(0,1^{\circ} \mathrm{C}\right.$ como valor medio en septiembre (Tabla 2). Los datos del suelo que llaman más la atención, por sus valores tan extremos, corresponden al nivel más superficial $(-5 \mathrm{~cm})$ donde la amplitud térmica alcanza los $48,2^{\circ} \mathrm{C}$, registrándose, además, las mínimas extremas absolutas más bajas de todo el perfil, $-19,6^{\circ} \mathrm{C}$ en invierno (diciembre), pero, también las más elevadas, $28,6^{\circ} \mathrm{C}$ en verano (agosto). La razón de este comportamiento debe estar asociada a la capacidad y rapidez de calentamientoenfriamiento que sufre este nivel superficial del suelo, controlado, estacionalmente, por la existencia de la cubierta nival, sobre todo, durante los periodos que limitan la larga estación fría invernal, pero ausente de ella en los meses centrales del verano.

\subsection{Días de helada, sin helada y con hielo-deshielo}

La Tabla 5 y la Figura 8 muestran el reparto porcentual anual de los días con temperatura negativa, positiva y positiva-negativa del aire y del suelo, lo que podría relacionarse, a grandes rasgos, como días con helada, sin helada, y con hielo-deshielo. Dos hechos destacan: el porcentaje de días con temperatura negativa crece entre el aire (39\%) y los $-150 \mathrm{~cm}$ de profundidad $(88,5 \%) \mathrm{y}$, por el contrario, decrecen en igual sentido por lo que respecta a los días con temperatura positiva $(33,7 \%$ del aire y $9,3 \%$ a $-150 \mathrm{~cm})$. En cuanto a los días en los que la temperatura oscila en torno a los $0^{\circ} \mathrm{C}$ los porcentajes resultan distorsionados en el nivel más profundo (2,2\%) frente a los $0,7 \%, 0,5 \%$ y $1,1 \%$ de los niveles $-100 \mathrm{~cm},-50 \mathrm{~cm}$ y $-20 \mathrm{~cm}$, respectivamente. A primera vista estos datos pudieran desconcertar, pero encuentran su lógica si se tiene en cuenta que aquello que se contabiliza es el cambio de signo en los valores registrados y en el caso del nivel $-150 \mathrm{~cm}$ éstos se encuentran muy próximos a los $0^{\circ} \mathrm{C}$, pues la amplitud térmica entre la media de las máximas extremas/media de las mínimas extremas es muy pequeña $\left(0,1^{\circ} \mathrm{C}\right)$, lo que viene a decir que tan sólo un incremento o disminución de décimas de grado ya puede implicar el cambio de signo en el valor registrado.

En cuanto al reparto estacional de los días de helada, sin helada y con hielo-deshielo en el comportamiento térmico del aire, la estación más equilibrada resulta el otoño, seguida de la primavera, mientras que las extremas son el verano y el invierno. Por lo que respecta al porcentaje de días de hielo-deshielo los valores máximos coinciden con las estaciones pre y postnivales, propiamente dichas $(32,2 \%$ en otoño y $39,6 \%$ en primavera). Como es lógico el máximo valor de días con temperatura positiva coincide con el verano $(93,2 \%)$, mientras aquellos con permanencia de valor negativo se dan en invierno $(72,2 \%)$. 


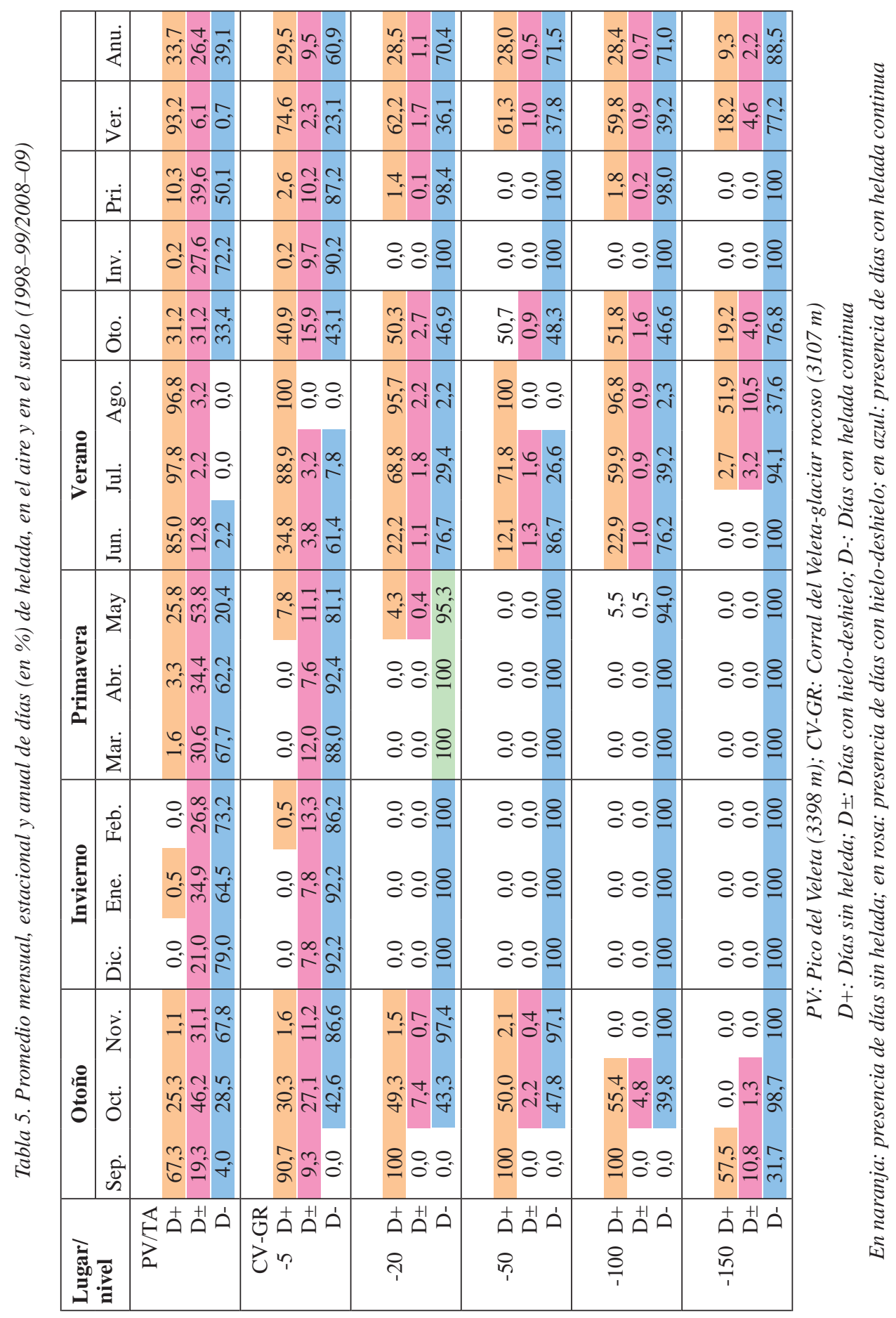




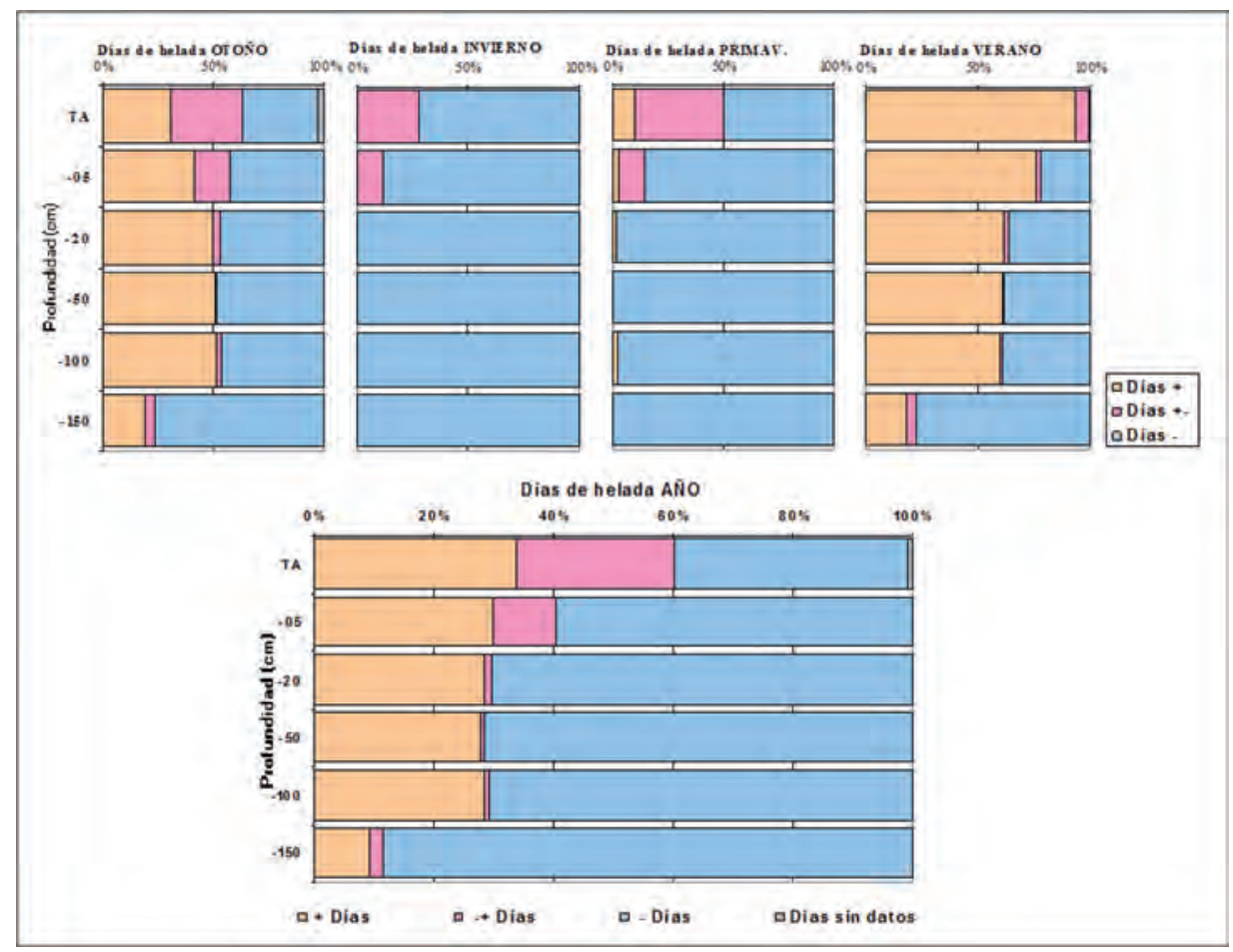

Figura 8. Distribución media estacional y anual del número de días (en \%) de helada en el aire y en el suelo (1998-99/2008-09).

En relación a la distribución del régimen térmico del suelo destaca, sobre todo, el elevado número de días de helada continua en superficie $(60,9 \%)$ aumentando paulatinamente en profundidad (88,5\%). Desde final de otoño y durante invierno-primavera el engelamiento es permanente a partir de $-20 \mathrm{~cm}$. Muy significativa es, también, la escasa presencia de días de hielo-deshielo (solo 9,5\% en superficie).

\subsection{Ciclos de hielo-deshielo}

Por su significado morfoclimático y su relación directa con la dinámica periglaciar que caracteriza las cumbres de Sierra Nevada, resulta del máximo interés tener conocimiento aproximado, también, del número de ciclos hielo-deshielo (dos cambios de fase térmica $=1$ ciclo) registrados a lo largo del año en el suelo, en contraste con los contabilizados en el aire (Tabla 6 y Figura 9). El centenar de ciclos presentes en el aire $(108,0)$ se reduce a un tercio en la superficie del suelo $(32,2)$. Por lo que respecta al aire los ciclos de hielo deshielo se mantienen a lo largo de todos los meses, siendo máximos en primavera $(38,3)$ y otoño $(36,1)$, seguidos del invierno $(28,0)$ y menores en verano $(5,7)$. 


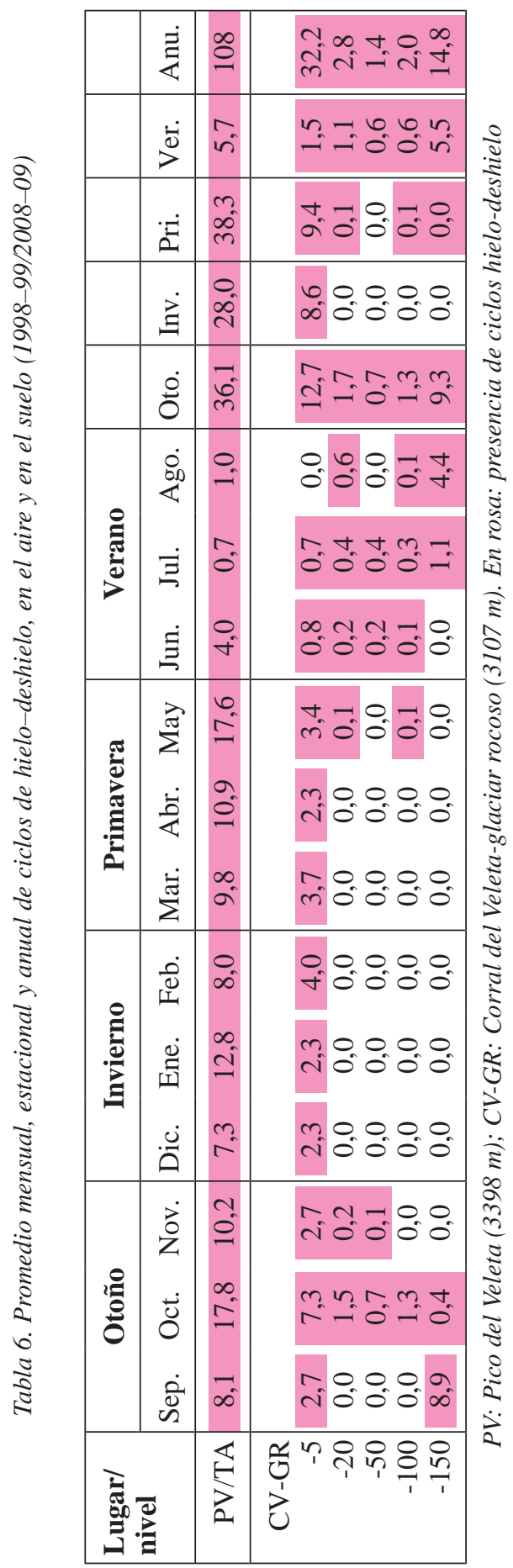




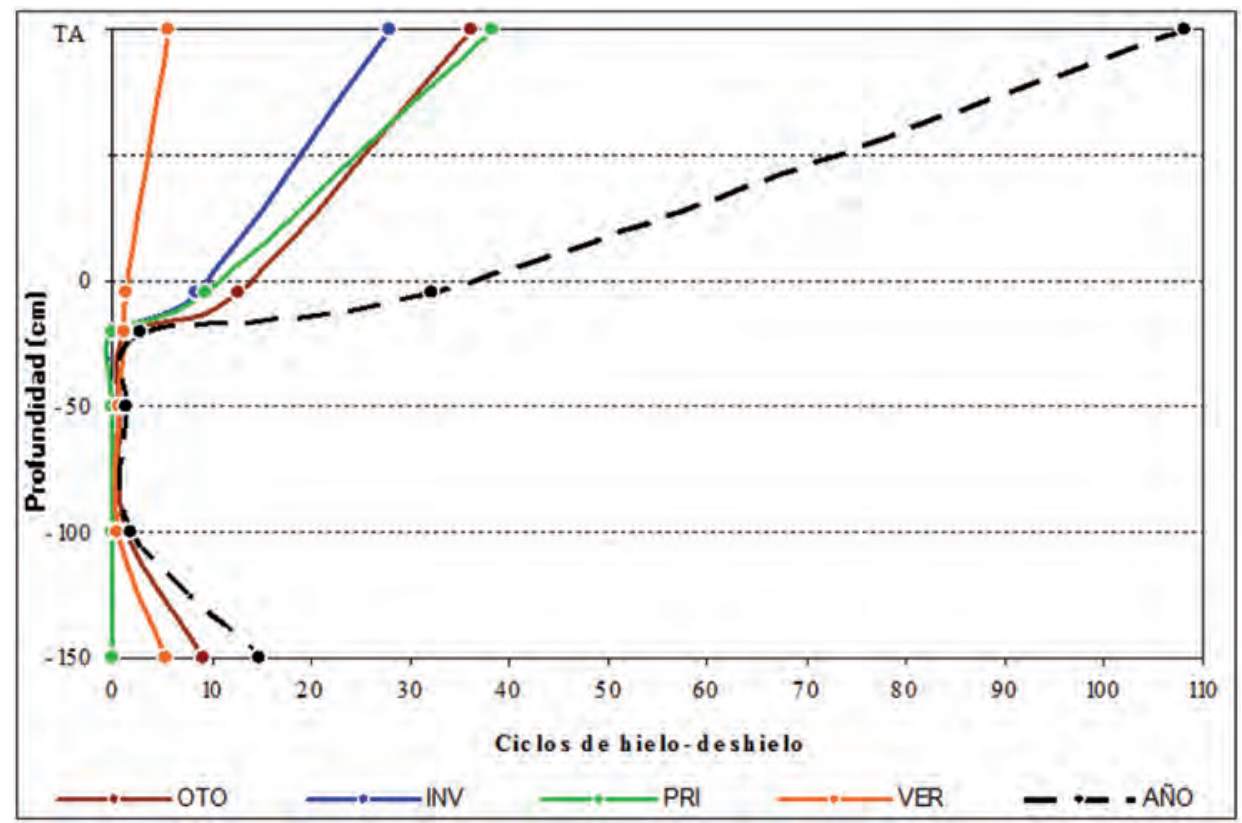

Figura 9. Promedio del número de ciclos de hielo-deshielo, estacional y anual, en el aire y en el suelo (1998-99/2008-09).

En cuanto a la distribución de valores de los ciclos de hielo-deshielo relativa al interior del suelo es variable: a $-5 \mathrm{~cm}, 32,2$ y a $-150 \mathrm{~cm}, 14,8$. Respecto a los niveles intermedios resultan más homogéneos, pues se sitúan en 2,8, 1,4 y 2,0 ciclos en los niveles de $-20 \mathrm{~cm},-50 \mathrm{~cm}$ y $-100 \mathrm{~cm}$, respectivamente. La ausencia de tales ciclos coincide con la estación fría, desde finales de otoño hasta inicios del verano, pues durante estos meses la temperatura se mantiene estable con valores negativos. Acerca del nivel $-150 \mathrm{~cm}$, con 14,8 ciclos al año, como ya se señaló en el apartado anterior (número de días de hielo-deshielo), el incremento que se detecta es atribuible a muy ligeras oscilaciones de pocas décimas en torno a $0^{\circ} \mathrm{C}$. Aún tratándose de ciclos "teóricos" o "potenciales", pues no consideramos por el momento su intensidad ni duración, su presencia a lo largo del perfil muestreado es baja.

\section{Análisis y discusión de resultados}

El seguimiento y control del comportamiento térmico del aire y, particularmente del suelo (capa activa) del glaciar rocoso del Corral del Veleta, ha mostrado un ritmo anual caracterizado por cuatro periodos diferenciados en duración temporal y rango de valores de temperatura: uno frío, otro de temperaturas moderadas y otros dos, de tránsito entre los anteriores. 


\subsection{Patrón de conducta térmico del suelo}

Los cuatro periodos térmicos reseñados se recogen en la Figura 10 y se refleja mejor su distribución en profundidad en la Figura 11. La información que se ofrece resulta de los valores medios para el conjunto de la serie analizada (1998/99-2008/09).

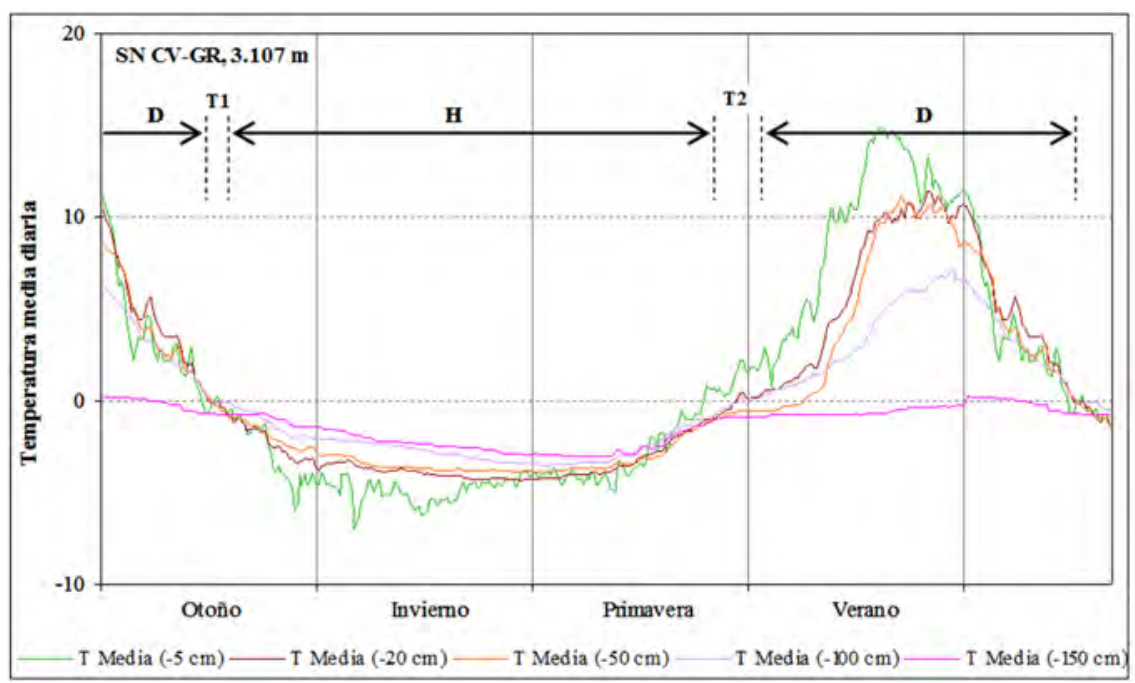

Figura 10. Evolución térmica media de los distintos niveles del suelo (patrón de comportamiento térmico), con indicación de los distintos periodos diferenciados (compárese con la Fig. 5). Los meses de septiembre y octubre aparecen repetidos al final del gráfico para facilitar la observación del periodo cálido (D).

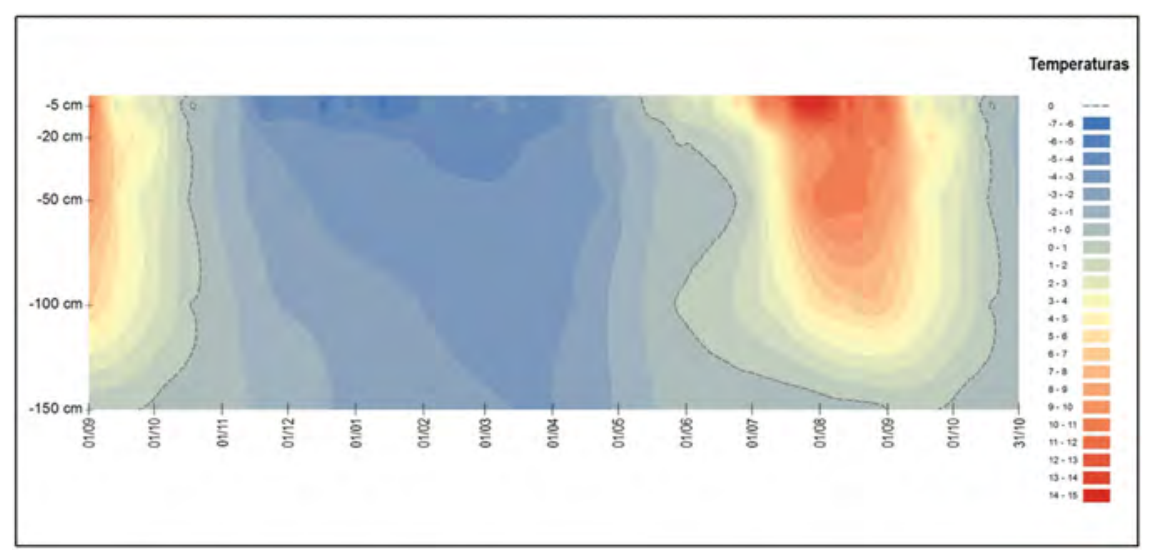

Figura 11. Distribución espacio-temporal de las temperaturas medias diarias del suelo (patrón de comportamiento térmico), mediante interpolación con kriging ordinario (compárese con la Fig. 6). Los meses de septiembre y octubre aparecen repetidos al final del gráfico para facilitar la observación del periodo cálido. 
Los periodos que definen el comportamiento térmico del suelo en el Corral del Veleta coinciden, en gran modo, con los propuestos por Delaloye (2004) en los Alpes suizos y, en menor medida, con los de González Trueba y Serrano (2010) para Picos de Europa.

El periodo frío resulta muy largo, de finales de octubre-noviembre hasta bien entrado junio. En total supone un valor medio de duración de 219 días. Durante ellos las temperaturas de superficie y de todo el perfil de suelo muestreado se sitúan en torno a los cero grados o por debajo de éste, aunque no se superan los $-3,6^{\circ} \mathrm{C}$ de promedio para todo el perfil.

El periodo de temperaturas moderadas (“cálidas") coincide de pleno con el verano y abarca un total de 92 días. Los valores medios anuales de temperaturas son positivos, aunque sin rebasar los $9,1^{\circ} \mathrm{C}$. A partir de la superficie las temperaturas decrecen registrándose puntualmente valores medios negativos en $10 \mathrm{~s}-150 \mathrm{~cm}\left(-0,6^{\circ} \mathrm{C}\right)$, aunque con alguna recuperación que puede superar ligeramente los $0^{\circ} \mathrm{C}$.

El tránsito entre los periodos anteriores incluye otros dos periodos temporales diferenciados por el cambio de signo en los valores registrados. Uno (T1), muy corto, donde el signo positivo del verano da paso a los negativos de la estación fría. Se inicia a finales de septiembre y concluye a principio de noviembre, que es cuando el manto nival tiende a permanecer estable en la superficie del suelo. Abarca en torno a 26 días. Durante su inicio las temperaturas están próximas a $\operatorname{los} 0^{\circ} \mathrm{C}$. Primero, con notable oscilación, luego, consolidándose y fijándose de manera estable en valores negativos. El episodio T2 resulta similar en duración y se desarrolla a lo largo de unos 28 días. Comienza apenas el manto nival tiende a permanecer inestable en el suelo, en torno a finales del mes de mayo. A inicios de julio, a excepción del nivel más profundo, el resto del perfil del suelo ya ofrece valores superiores a los $3^{\circ} \mathrm{C}$. Así como el episodio $\mathrm{T} 1$ es de marcado ritmo rápido, el T2 resulta más pausado.

\subsection{El cometido de la nieve en el suelo}

El comportamiento térmico del suelo durante el largo periodo frío y aquél otro corto de temperaturas moderadas del verano está directamente determinado por la presencia-ausencia de nieve. La nieve en las cumbres de Sierra Nevada y, en concreto, en el Corral del Veleta ya permanece estable a mediados de noviembre y así se mantiene, aunque aumentando de espesor, hasta finales de mayo en que inicia su fusión. En agosto, habitualmente, la nieve en el suelo ha desaparecido, salvo aquellos años en que ésta ha sido muy copiosa, apareciendo entonces neveros dispersos.

La cubierta nival en el suelo y su duración en el tiempo explica el prolongado valor negativo de las temperaturas así como la reducida amplitud térmica que se detecta en los distintos niveles muestreados durante la larga estación fría. También se comprende que, mermada la nieve del suelo o desaparecida totalmente del mismo, hecho que empieza a darse progresivamente y de forma muy frecuente a partir de finales de mayo-primeras 
semanas de junio, la radiación térmica externa afecta directamente la superficie del suelo desnudo y alcanza rápidamente sus capas internas propiciando temperaturas positivas en todo el perfil (valores medios de la estación estival superiores a los $3^{\circ} \mathrm{C}$ en $\operatorname{los}-100 \mathrm{~cm}$ y a $\operatorname{los} 5^{\circ} \mathrm{C}$ por encima de los $-50 \mathrm{~cm}$ ). En cuanto al nivel de $-150 \mathrm{~cm}$ la temperatura media, si bien se refleja negativa $\left(-0,6^{\circ} \mathrm{C}\right)$, el registro diario real refleja un ritmo de valores frecuentes en torno a los cero grados. Cuando la nieve no desaparece durante el corto estío, como sucedió en 2000-01 y recientemente en 2009-10, las temperaturas del suelo, probablemente, no puedan alcanzar valores positivos debido al efecto aislante que genera el manto nival respecto a la radiación externa. Los registros que obtengamos próximamente correspondientes al periodo 2009-11 nos darán información de ello.

\subsection{Ritmo térmico y degradación del techo de las masas heladas subyacentes}

Desde la campaña 2001-02 disponemos de información relativa a la degradación del techo de las masas heladas (hielos glaciares y permafrost) que se incluyen en el interior del glaciar rocoso monitorizado térmicamente. Esta degradación se manifiesta por colapsos variables del manto detrítico. La causa de ellos es el proceso de inestabilidad que sufre el techo helado en el que reposa el paquete de bloques del glaciar rocoso, motivado, presumiblemente, por un régimen térmico capaz de generar fusión de la referida masa helada. Los resultados obtenidos de esta degradación vienen a establecer la relación directa entre cubierta nival, régimen térmico del suelo y magnitud del colapso. Debe tratarse de procesos físicos encadenados en el tiempo. El colapso está supeditado a la eficacia térmica en el interior del suelo, y ésta al control que pueda ejercer la nieve sobre la radiación externa durante el periodo de temperaturas moderadas del verano. La Tabla 7 refleja fielmente estos hechos. Los datos corresponden al tramo medio del glaciar rocoso, que es donde se llevan a cabo los registros térmicos analizados. Los diferentes controles realizados se obtuvieron durante la última semana del mes de agosto de los años respectivos (2004 y 2005) (Gómez Ortiz et al., 2008).

Tabla 7. Relación entre cubierta nival, régimen térmico del suelo y magnitud del colapso de la masa helada

\begin{tabular}{|c|c|c|c|c|}
\hline Periodo* & $\begin{array}{c}\text { Recubrimiento } \\
\text { nival tercio } \\
\text { oriental CV } \\
\text { (\% de suelo } \\
\text { cubierto) }\end{array}$ & $\begin{array}{c}\text { Duración } \\
\text { temperatura } \\
\text { positiva en el } \\
\text { Suelo* (días) }\end{array}$ & $\begin{array}{c}\text { Hundimiento } \\
\text { medio superficie } \\
\text { GR }(\mathbf{c m})\end{array}$ & $\begin{array}{c}\text { Pérdida de } \\
\text { volumen de } \\
\text { masa helada** } \\
\left(\mathbf{m}^{\mathbf{3}}\right)\end{array}$ \\
\hline $2003-04$ & $>50$ & 25 & 7,1 & 270,8 \\
\hline $2004-05$ & 0 & 105 & 55,6 & 2121,1 \\
\hline
\end{tabular}

CV: Corral del Veleta; GR: glaciar rocoso

* mayo-agosto; ** se refiere al conjunto del glaciar rocoso, suponiendo movimiento y espesor homogéneo. 


\section{Conclusiones}

El análisis del comportamiento térmico del suelo del glaciar rocoso del Corral del Veleta viene a mostrarnos la particularidad morfotopográfica y glaciológica de este enclave de Sierra Nevada, pues es el único que aún conserva restos de masas heladas heredades de periodos más fríos que la actualidad. Los controles nivológicos realizados (Tanarro et al., 2010) junto a los datos térmicos obtenidos a lo largo del registro casi continuo 1998-99/2008-09, permiten otorgar a la presencia de nieve en el suelo un cometido determinante en el precario mantenimiento de las masas heladas relictas, aunque en evidente proceso de degradación. Este proceso es repetitivo y se lleva a cabo durante el corto periodo de temperaturas moderadas (verano) siempre que falte la nieve en el suelo pues la temperatura del aire es capaz de penetrar en la capa activa y alcanzar el techo de los niveles helados propiciando temperaturas favorables para su degradación y la consiguiente inestabilidad y colapso del paquete de bloques suprayacente (Fig. 11).

Asociado a este proceso degradativo de las masas heladas encerradas en el interior del glaciar rocoso hay que entender, también, el dinamismo de éste. Los resultados del periodo 2001-2009 en relación a su desplazamiento planar (a favor de la pendiente) y vertical (hundimiento-colapso) indican un acusado predominio de este último movimiento, muy diferente en valor a lo que ocurre en glaciares rocosos de montañas de latitudes cercanas (Pirineo central y Alpes austríacos) (Sanjosé et al., 2010). Los resultados comparativos de la Tabla 8, referidos al periodo 2002-2009 y al tramo medio de cada uno de los glaciares rocosos analizados, vienen a demostrarlo destacándose, sobre todo, la magnitud del desplazamiento vertical que se registra en el Corral del Veleta, lo cual sugiere pensar que se trata de un glaciar rocoso decrépito que tiende a su inmovilización por progresiva reducción de sus masas heladas basales y adyacentes (hielo glaciar y permafrost), como respuesta a la eficacia periódica de la temperatura interna del suelo.

Tabla 8. Comparación del desplazamiento observado en el tramo medio de los glaciares rocosos de Doesen, Posets y Corral del Veleta (periodo 2002-2009)

\begin{tabular}{|l|c|c|c|c|c|c|}
\hline $\begin{array}{c}\text { Glaciar } \\
\text { rocoso }\end{array}$ & Localización & $\begin{array}{c}\text { Cotas } \\
\text { S.n.m. } \\
(\mathbf{m})\end{array}$ & Macizo & $\begin{array}{c}\text { Periodo de } \\
\text { observación }\end{array}$ & $\begin{array}{c}\text { Movimiento } \\
\text { planar = } \\
\text { avance (cm) }\end{array}$ & $\begin{array}{c}\text { Movimiento } \\
\text { vertical = } \\
\text { hundimiento } \\
\text { (cm) }\end{array}$ \\
\hline Doesen & $\begin{array}{c}46^{\circ} 59^{\prime} 12^{\prime \prime} \mathrm{N} \\
13^{\circ} 17^{\prime} 08^{\prime \prime} \mathrm{E}\end{array}$ & $2339-2650$ & $\begin{array}{c}\text { Alpes } \\
\text { austriacos }\end{array}$ & $2002-2009$ & 32,9 & 10,5 \\
\hline Posets & $\begin{array}{c}42^{\circ} 39^{\prime} 32^{\prime \prime} \mathrm{N} \\
0^{\circ} 26^{\prime} 49^{\prime \prime} \mathrm{E}\end{array}$ & $2995-3060$ & $\begin{array}{c}\text { Pirineo } \\
\text { Central }\end{array}$ & $2002-2009$ & 15,6 & 9,9 \\
\hline $\begin{array}{l}\text { C. de } \\
\text { Veleta }\end{array}$ & $\begin{array}{c}37^{\circ} 03^{\prime} 33^{\prime \prime} \mathrm{N} \\
3^{\circ} 21^{\prime} 47^{\prime \prime} \mathrm{W}\end{array}$ & $3090-3110$ & $\begin{array}{c}\text { Sierra } \\
\text { Nevada }\end{array}$ & $2002-2009$ & 5,9 & 34,7 \\
\hline
\end{tabular}




\section{Agradecimientos}

Al proyecto de investigación 018/2007 del Organismo Autónomo Parques Nacionales (MMA). También al CSO2009-06961 del Ministerio de Ciencia e Innovación. Este estudio se enmarca en las actividades investigadoras del Grupo de Investigación Consolidado SGR2009-0898 Paisatge i paleoambients a la muntanya mediterrània de la Generalitat de Catalunya (Universitat de Barcelona).

\section{Referencias bibliográficas}

Andrés De Pablo, N., Palacios, D., Úbeda Palengue, J., Alcalá Reygosa, J., (2010a). Relación entre las anomalías geotérmicas y la ausencia de formas glaciares y periglaciares en el volcán el Misti (sur del Perú). Boletín de la AGE (admitido).

Andrés de Pablo, N., Palacios, D., Úbeda Palenque, J., Alcalá Reygosa, J., (2010b). Distribución del medio periglacial y del permafrost en un volcán tropical extinto: Nevado de Chachani (sur de Perú). Scripta Nova. Revista electrónica de Geografía y Ciencias Sociales (admitido).

Andrés de Pablo, N., Palacios, D., (2010c). Cubierta nival y distribución de la temperatura en el suelo en las cumbres de la Sierra de Guadarrama. Cuadernos de Investigación Geográfica, 36 (2): 7-36.

CALlendar, H.L., (1897). Observations of Soil Temperatures with electrical resistance thermometers. Trans. Roy. Soc. Canada, 2nd series, vol. III, pp. 31-49.

Carrera Gómez, P., Mikkan, R., Pérez Alberti, A., Valcárcel Díaz, M., Blanco ChAO, R., López Bedoya, J., (2008). Avance de resultados sobre el estudio del régimen térmico del suelo en un sector de la alta montaña andina (Parque Provincial Aconcagua, Andes de Mendoza. Argentina). Trabajos de Geomorfología en España (2006-2008), SEG-Universidad de Cádiz, pp. 233-236, Cádiz.

ChuecA, J., Julián, A., (2010). Caracterización térmica del suelo en el circo del Aneto (Pirineo central aragonés): cartografía de variaciones estacionales. En: Ambientes periglaciares y permafrost y variabilidad climática (Blanco, J.J., de Pablo, M.A., Ramos, M., Eds.), Servicio de Publicaciones Universidad de Alcalá de Henares, pp. 55-60, Alcalá de Henares.

Delaloye, R., (2004). Contribution à l'étude du pergélisol de montagne en zone marginale. GeoFocus, Vol. 10, Université de Fribourg, 240 pp., Fribourg.

Gómez Ortiz, A., Salvador Franch, F., Schulte, L., SAnjosé, J.J., Palacios, D., (2008). Evolución morfodinámica de un enclave montañoso recién deglaciado: el caso del Corral del Veleta (Sierra Nevada), ¿consecuencia del cambio climático? Scripta Nova. Revista electrónica de Geografía y Ciencias Sociales, 12(270): 26. 
Gómez Ortiz, A., Palacios, D., Schulte, L., Salvador Franch, F., Plana CasTELLVÍ, J.A., (2009a). Evidences from historical documents of landscape evolution after Little Ice Age of a mediterranean high mountain area, Sierra Nevada, Spain (Eighteenth to Twentieth Centurias). Geografiska Annaler, 91A(4): 279-289.

Gómez Ortiz, A., Oliva, M., SAlvador Franch, F., (2009b). Registros naturales y documentación histórica relativos a la Pequeña Edad del Hielo en las cumbres de Sierra Nevada. En: El sector central de las Béticas: una visión desde la geografía física (Gómez Zotano, J., Ortega Alba, F., Eds.), Ed. Universidad de Granada, pp. 245-259, Granada.

GonzÁlez Trueba, J.J., Serrano, E., (2010). La nieve en los Picos de Europa: implicaciones geomorfológicas y ambientales. Cuadernos de Investigación Geográfica, 36 (2): $61-84$.

HaEberli, W., (1973). Die Basis Temperatur der winterlichen Schneedecke als möglicher Indikator für die Verbreitung von Permafrost. Zeitschrift für Gletscherkunde und Glazialgeologie, 9 (1-2): 221-227.

Hoelzle, M., Wegmann, M., Krummenacher, B., (1999). Miniature Temperature Dataloggers for Mapping and Monitoring of Permafrost in High Mountain Areas: First Experience from the Swiss Alps. Permafrost and Periglacial Processes, 10: 113-124.

KING, L., (1990). Soil and rock temperatures in discontinuous permafrost: Gornergrat and Unterrothorn, Wallis, Swiss Alps. Permafrost and Periglacial Processes, 32: 1797-1810.

Marcos, J., Palacios, D., (2004). Efectos de la nieve y la temperatura del suelo en la actividad geomorfológico: primeros resultados de su monitorización en la Sierra de Guadarrama, España. Boletín de la Real Sociedad Española de Historia Natural, 99 (1-4): 25-36.

Oliva, M., Gómez OrTiz, A., (2010). Monitorización dinámica y control térmico en geoformas solifluidales del piso crionival de Sierra Nevada. En: Ambientes periglaciares y permafrost y variabilidad climática (Blanco, J.J., de Pablo, M.A., Ramos, M., Eds.), Servicio de Publicaciones Universidad de Alcalá de Henares, pp. 29-34, Alcalá de Henares.

Palacios, D., Andrés de Pablo, N., Úbeda Palenque, J., Alcalá Reygosa, J., (2009). Permafrost and Periglacial Activity Distribution and Geothermal Anomalies in the Chachani and El Misti Volcanoes (Southern Peru). Geophysical Research Abstracts, Vol. 11. EGU 2009-8014-2. EGU General Assembly.

Ramos, M., Gómez Ortiz, A., SAlvador Franch, F., Schulte, L., (1998). Evolución térmica de la capa activa en la estación geomorfológico de la Planicie La Feixa-La Màniga, 2150 m (macizo de Calmquerdós. Pirineo Oriental). En: Procesos biofísicos actuales en medios fríos (Gómez-Ortiz, A., Salvador-Franch, F., Schulte, L., GarcíaNavarro, A., Eds.), Universitat de Barcelona, pp. 73-97, Barcelona. 
Ramos, M., Gómez Ortiz, A., Palacios, D., Tanarro, L.M., Salvador Franch, F., CRespo, F., BREtón, L., (2002). Método térmico para el estudio de la distribución de permafrost en Sierra Nevada. En: Periglaciarismo en montaña y altas latitudes (Serrano Cañadas, E., García de Celis, A., Eds.), Universidad de Valladolid, pp. 91-123, Valladolid.

RAMOS, M., VIEIRA, G., (2004). Variabilidad térmica de la capa activa y evaluación de la energía perdida por el suelo durante el proceso de congelación en la Isla Livingston (Antártida). Inviernos 2000, 2001 y 2002. Boletín de la Real Sociedad Española de Historia Natural. Sección Geología, 99 (1-4): 83-92.

Santos GonzÁlez, J., González Gutiérrez, B., Gómez Villar, A., Redondo VEGA, J.M., (2009). Ground thermal regime in the vicinity of relict rock glaciers (Cantabrian mountains, NW Spain). Finisterra, XLIV, 87: 35-44.

Salvador Franch, F., Gómez Ortiz, A., Palacios, D., (2010). Comportamiento térmico del suelo en un enclave de alta montaña mediterránea con permafrost residual: Corral del Veleta (Sierra Nevada, Granada. España). En: Ambientes periglaciares, permafrost y variabilidad climática (Blanco, J.J., de Pablo, M.A., Ramos, M., Eds.). Servicio de Publicaciones Universidad de Alcalá de Henares, pp. 61-68, Alcalá de Henares.

Sanjosé, J.J., Kaufmann, V., Gómez Ortiz, A., Serrano, E., Atkinson, A., SalvaDOR FrANCH, F., GonZÁlEZ TruebA, J.J., (2010). Técnicas geomáticas aplicadas al control de los glaciares rocosos. Comparación de los glaciares rocosos de Doesen (Alpes), Posets (Pirineos) y Corral del Veleta (Sierra Nevada). Revista Cartográfica (en prensa).

Serrano, E., SAnjosé, J.J., Agudo, C., (2006). Rock glacier dynamics in a marginal periglacial high mountain environment: Flow movement (1991-2000) and structure of the Argualas rock glacier, the Pyrenees. Geomorphology, 74: 285-296.

Serrano, E., Sanjosé, J.J., GonzÁlez Trueba, J.J., (2010). Rock glacier dynamics in marginal periglacial environments. Earth Surface Processes and Landforms, 35: $1302-1314$.

Tanarro, L.M., Palacios, D., Zamorano Orozco, J.J., Gómez Ortiz, A., (2010). Cubierta nival, permafrost y formación de flujos superficiales en un talud detrítico de alta montaña (Corral del Veleta, Sierra nevada, España). Cuadernos de Investigación Geográfica, 36 (2): 39-59.

VIEIRA, G., MoRA, C., RAmos, M., (2003). Ground temperature regimes and geomorphological implications in a mediterranean mountain (Serra da Estrela, Portugal). Geomorphology, 52: 57-72. 